\title{
ACCESSIBLE PATH FINDING FOR HISTORIC URBAN ENVIRONMENTS: FEATURE EXTRACTION AND VECTORIZATION FROM POINT CLOUDS
}

\author{
D. Treccani ${ }^{1,2, *}$ L. Díaz-Vilariño ${ }^{1}$, A. Adami $^{2}$ \\ ${ }^{1}$ Universidade de Vigo, CINTECX, GeoTECH group, 36310 Vigo, Spain - (daniele.treccani, lucia)@ uvigo.es \\ ${ }^{2}$ He.Su.Tech. group, MantovaLab, Dept. of Architecture Built environment and Construction engineering (ABC) \\ Politecnico di Milano, 46100 Mantua, Italy - (daniele.treccani, andrea.adami)@ polimi.it
}

\section{Commission II}

KEY WORDS: Semantic segmentation, Accessibility, Sidewalk inventory, Point cloud processing, Cultural heritage, Vectorization, QGIS, Network analysis.

\begin{abstract}
:
Sidewalk inventory is a topic whose importance is increasing together with the widespread use of smart city management. In order to manage the city properly and to make informed decisions, it is necessary to know the real conditions of the city. Furthermore, when planning and calculating cultural routes within the city, these routes must take into account the specific needs of all users. Therefore, it is important to know the conditions of the city's sidewalk network and also their physical and geometrical characteristics. Typically, sidewalk network are generated basing on existing cartographic data, and sidewalk attributes are gathered through crowdsourcing. In this paper, the sidewalk network of an historic city was produced starting from point cloud data. The point cloud was semantically segmented in "roads" and "sidewalks", and then the cluster of points of sidewalks surfaces were used to compute sidewalk attributes and to generate a vector layer composed of nodes and edges. The vector layer was then used to compute accessible paths between Points of Interest, using QGIS. The tests made on a real case study, the historic city and UNESCO site of Sabbioneta (Italy), shows a vectorization accuracy of $98.7 \%$. In future, the vector layers and the computed paths could be used to generate maps for city planners, and to develop web or mobile phones routing apps.
\end{abstract}

\section{INTRODUCTION}

Sidewalk inventory is a process related to several aspects of the administration of a city. In fact, for a correct planning and for a proper and informed decision making process related to accessibility management, a prior knowledge of the current condition of the urban environment is required. Moreover, when dealing with a historic city, the predisposition of accessible routes and pedestrian paths connecting Points of Interest (PoI) inside the city is an important process that should take in consideration the conditions and characteristics of the sidewalk network.

Furthermore, for tourism management, the predisposition of routes is important, and that should be done taking in consideration also the design of inclusive paths which can be easily accessed and exploited by all kind of users. Considering legislative framework about physical accessibility and architectural barriers (e.g., for Italy, law DM 236/1989 and DPR 503/1996) and in light of broader issues coming also from practice (Marconcini et al., 2021) it is possible to define some requirements that a sidewalk should have to be considered accessible. For example, the Italian law foresees: a minimum width of $90 \mathrm{~cm}$, level difference lower than $2.5 \mathrm{~cm}$, and a transverse slope lower than $1 \%$.

In this context, a detailed map of the existing sidewalks, together with their geometric attributes, is a proper basis for an informed decision making in city planning and also for the organization and the design of accessible routes inside the city. There are several ways to reach the purpose of creating a sidewalk network to manage physical accessibility of a city, and a map is usually the most effective starting point. In order to

\footnotetext{
* Corresponding author
}

produce this kind of maps, the use of crowdsourcing (Saha et al., 2019) or the employment of questionnaires completed by experienced technicians who have visited the site are common popular methods, but they are not immune to subjective opinions. On the other hand, the use of Information Communication Technology (ICT) tools that exploit rules and algorithms can be considered more objective (Marconcini and Pracchi, 2019).

Facing this topic on an historic urban environment, the management and calculation of accessible routes connecting two POIs is made more complex by the street framework of the historic city itself. Typical conditions are: narrow streets, a high number of intersections, roads temporarily closed to traffic and completely used by pedestrians, the presence or absence of zebra crossings, sidewalks of different widths, and urban pavements in various materials. For all these reasons, the optimal starting point is a map that correctly stores the geometric attributes of sidewalks and streets, so that the calculated route can take into account either the limitations actually present in the urban fabric, and the rules and regulations of urban accessibility.

Sidewalk network analysis is a topic investigated in literature usually referring to modern and regular road frameworks. The starting point come from various sources (point clouds, satellite images, cadastral maps), but the use of vector files and Geographical Information Systems (GIS) is the most common. Physical accessibility is typically checked punctually (on a specific sidewalk) and not globally (along the entire route). The work presented in this paper is based on a previously classified point cloud, and method is implemented for the automatic extraction of sidewalks geometric attributes and the vectorization of the sidewalk spatial position, forming a network. Then, the sidewalk network is leveraged for routing simulations taking in 


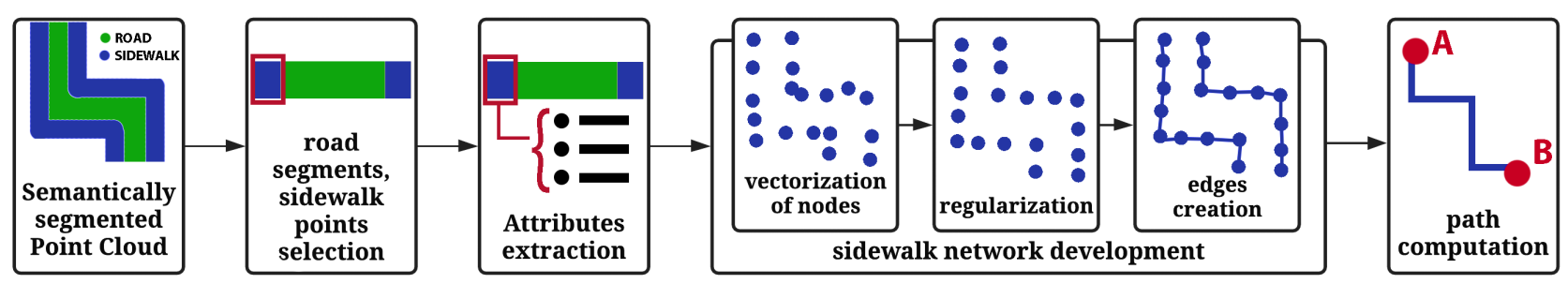

Figure 1. Workflow of the method. Starting from an urban environment semantically segmented point cloud, sidewalk points were extracted and used to compute accessibility attributes, and then are converted to a vector layer with nodes and edges to be used for path computations.

consideration some real life scenarios that may happen in a historic city. The method is tested on a historic city located in Northern Italy, Sabbioneta, which, together with Mantova is a UNESCO site since 2008 .

The paper is organized as follows. In section 2, Related works, existing literature is analyzed and existing methods dealing with route computation and sidewalk analysis are presented and compared. Section 3, Materials and method, describes the case study selected and the method, from the extraction of sidewalks geometric attributes to their vectorization and the analysis on the network. Section 4, Results, presents the resulting data on the case study, and the last section outline the conclusion and the further development and works.

\section{RELATED WORKS}

The topic of sidewalk network analysis is usually treated in literature starting from existing vector layer provided by municipalities, cadastral offices, or other agencies, and implementing it to compute accessible routes considering the needs of different users. An example in this sense is the work of (Verde et al., 2021), which is based on a GIS map of the streets of the historic centre, made available by the City Council. Thanks to the help of two institutions of visually impaired people, the roads were classified according to their accessibility, and a mobile phone application was developed to help people planning their routes inside the city according to their needs. Another example is the work of ( $\mathrm{Li}$ et al., 2018), where parcel polygons data and roadway centerline data were used to build a sidewalk network database. In their tests, authors reconstructed sidewalk nodes and edges taking in consideration also intersections and crosswalks, with the purpose of generating a fully connected network. Sidewalk attributes were then collected using specific Android- and IOS-compatible smartphone and tablet applications and then stored inside the database. The result was a GIS database useful for producing maps and computing pedestrian routes within the city.

In previous works, the starting point of the methods were preexisting GIS dataset, which were elaborated and enriched with information collected elsewhere, in order to reach the purpose. Another approach is to work directly on the point cloud, acquired with a Mobile Laser Scanning (MLS) system. This is the case of (Hou and Ai, 2020), where authors used a customized deep neural network to semantically segment a MLS point cloud, integrating a stripe-based sidewalk extraction algorithm. The resulting sidewalk information were then compared with accessibility compliance to asses the network condition.

Another possibility is to combine dataset coming from different sources, to compute optimal routes. This is the case of (Luaces et al., 2021), where authors used data from Open Street Map (OSM), added information by processing MLS point clouds, detected obstacles using crowdsourcing and identified accessibility issues analysing social networks data. These data were then used to produce an application able to compute optimal routes considering different needs.

Unlike what has been seen in the literature, in this paper the method presented is based on a point cloud processing approach and is extensively applied on a historic city, tackling all the peculiarities on an urban environment that is stratified and organized to cope with restriction and constraints that comes from the historic city framework itself.

\section{MATERIALS AND METHOD}

The method starts by a classified point cloud. The semantic segmentation implemented an heuristic method based on point similarities and topological information (Treccani et al., 2021). The dataset was a point cloud of a historic urban environment already classified with, among the others, a label "sidewalk" for the points pertaining to sidewalks surfaces. The point cloud was acquired using a MLS system and the trajectory was known.

Since sidewalks are the interesting urban element for this paper, only points of label "sidewalks" were selected and the point cloud of the whole city was subdivided in small portion following the roadway line, extracting and analysing a segment of 2 metres length at a time. Then, from each sidewalk segment, some geometric attributes were computed, that have to be considered for an accessibility map. Each sidewalk segment was then stored into a vector layer and used as network for routes computation implementing processes already existing in the software QGIS (Pella and Ose, 2018). A summary of the workflow is presented in Figure 1.

\subsection{Case study:UNESCO site of Sabbioneta}

The case study selected for the tests presented in this paper is the historic city of Sabbioneta, built between 1556 and 1591 by Vespasiano Gonzaga, following the "ideal city" principles. The city present a chessboard scheme with a main road that connect the two fortified doors of the city, located on two opposite sides. The road framework is composed by roads with different width, from very narrow to very large; and different paving materials were used, bricks and stone for sidewalks, cobblestone and sampietrini for roadway and squares. The point clouds dataset used for this test was acquired in 2020 with a Leica Pegasus:Two, and processed in order to perform a semantic segmentation whose result is a point cloud of all the ground surfaces, with all the points classified in "road surface" and "sidewalk surface". 


\subsection{Sidewalk attribute extraction}

The point cloud was subdivided in sub-clouds using Bounding Boxes created from two-metre-long segments extracted from the trajectory line of the acquisition instrument. In this paper the sub-clouds will be called "segments". For each segment points of sidewalks on left and right side were analysed with the purpose of computing some geometric attributes of the sidewalks itself. The attributes are summarized by Figure 2 and here described in details:

- width: assuming that the sidewalk width is uniform along the analyzed segment, and that the sidewalk is parallel to the roadway line, width was computed by exploiting the difference between the further and closer point of the sidewalk respect the roadway line (taking care of eventual noisy points); the value was then rounded to the closer 5 centimetres

- sidewalk-road relative elevation: if present, relative elevation was computed by exploiting the difference between the average Z-coordinate value of sidewalk points and road points in the proximity of sidewalks;

- transverse and longitudinal slopes: after having computed the Principal Component Analysis (PCA) of sidewalk points, the slopes of the longer eigenvectors represented the slope of the sidewalk itself;

- main material of the paving surface: implementing a machine learning tool it was possible to predict the paving material; for the case study tested the pavings were only of two types: bricks or stone.

\subsection{Sidewalk network vectorization}

Firstly, each sidewalk cluster of points was converted in a vector format in order to produce and realize a vector network. To do so, it was decided to represent each cluster as a single point (i.e. node), and the computed central point (average XYZcoordinates) of each cluster of points was used as the representative for the sidewalk segment itself and sidewalk attributes were linked to that point.

At this stage, all sidewalks were represented by several points, almost aligned and parallel to the roadway. A refinement was here necessary, so considering the contextual information, the points were slightly moved in order to obtain a more regularized result. To do so (see Fig. 3) the three types of roads crossings existing in the case study were analysed, named $\mathrm{X}$ crossing, $\mathrm{T}$ crossing and corner, usually at 90 degrees, and so named Lcrossing. In order to define the position of the crossings, the OSM dataset was used; in specific, the road lines and their vertexes were exploited to define crossing position and their type. Then for each crossing type, the points on the vector layer were selected and using a least square method, the best fit line was computed and the points were slightly moved on $\mathrm{X}$ and $\mathrm{Y}$ to finally be on the computed line.

As last, consecutive points (i.e. nodes) were linked together by lines (i.e. edges) forming a network. To easily compute the routes, the same attributes stored in the nodes are passed to the edges. The resulting vector network represents a high level of information for sidewalks because it has punctual information of sidewalk attributes each 2 metres along the sidewalk framework of the city. A final manual refinement was then foreseen;
Attribute: width

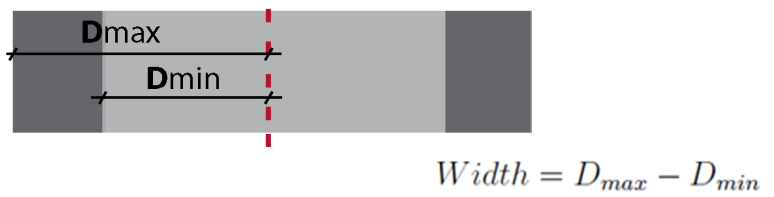

Attribute: elevation difference

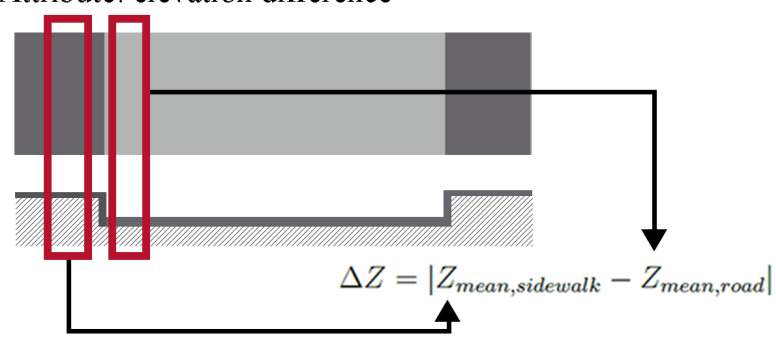

Attribute: slopes

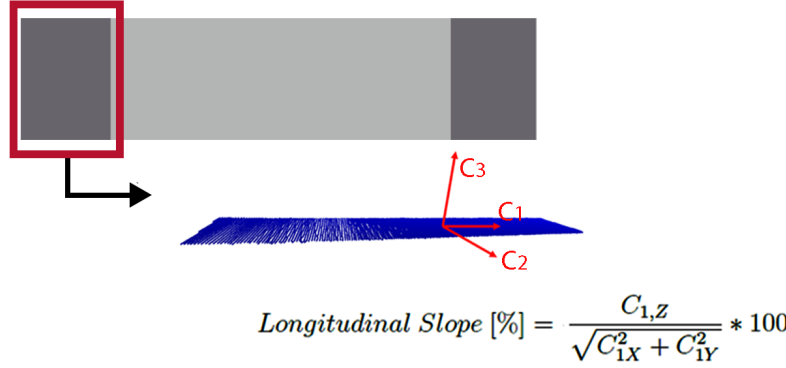

Attribute: paving material

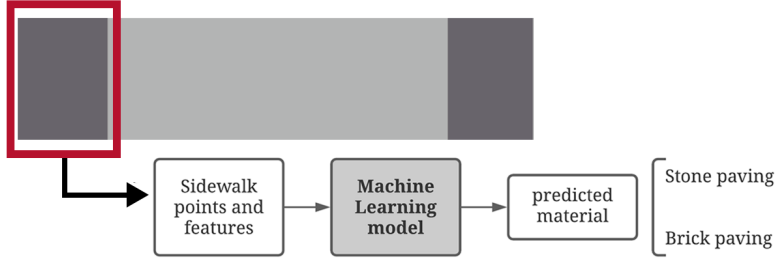

Figure 2. Explanation of all the sidewalk geometric attributes extracted from the point cloud and how they were computed.

for example the zebra crossings were added manually. In fact, even if in literature many zebra crossing recognition methodology exist, in this paper an automatic zebra crossing detection is not implemented, also because zebra crossing in the historic city are not identifiable by street sign marks, but, for example using a different flooring respect the roadway. At the moment they were added manually to the vector network.

Furthermore, another consideration should be made, in the case study selected there are only few (actually only 3 ) zebra crossing and in the majority of the city pedestrian are allowed to cross the road in every position. To recreate this behaviour a second vector network was developed, by connecting not only the closer sidewalk points, but also connecting points across the road.

\subsection{Path computation}

The vectorized sidewalk network stored in a shapefile was imported in QGIS. Two types of networks were imported: a first one representing only the sidewalks, that can be used to prepare thematic map that shows sidewalks attributes' data; and 


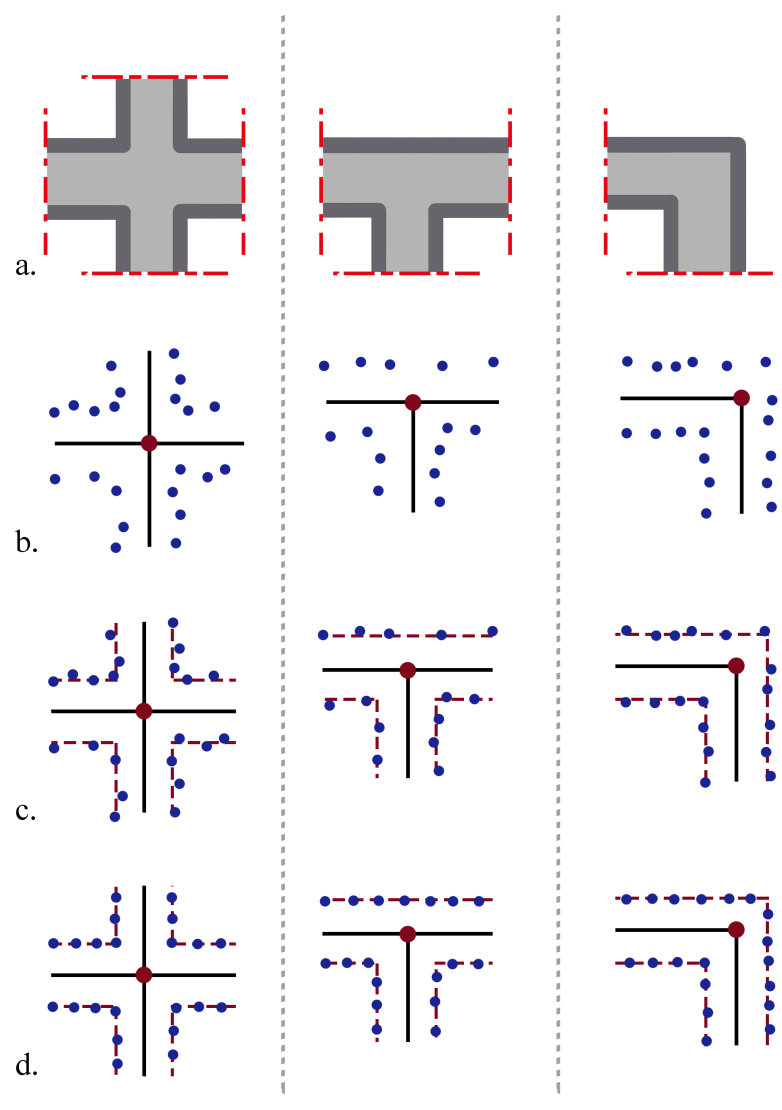

Figure 3. Steps for the regularization of vectorized sidewalk nodes, according to three types of roads crossings (a): X-shape, T-shape, and L-shaped corners. From top to bottom: firstly (b) the type of crossing was identified, then (c) reference lines were computed and then (d) points were slightly moved in order to lie on those lines.

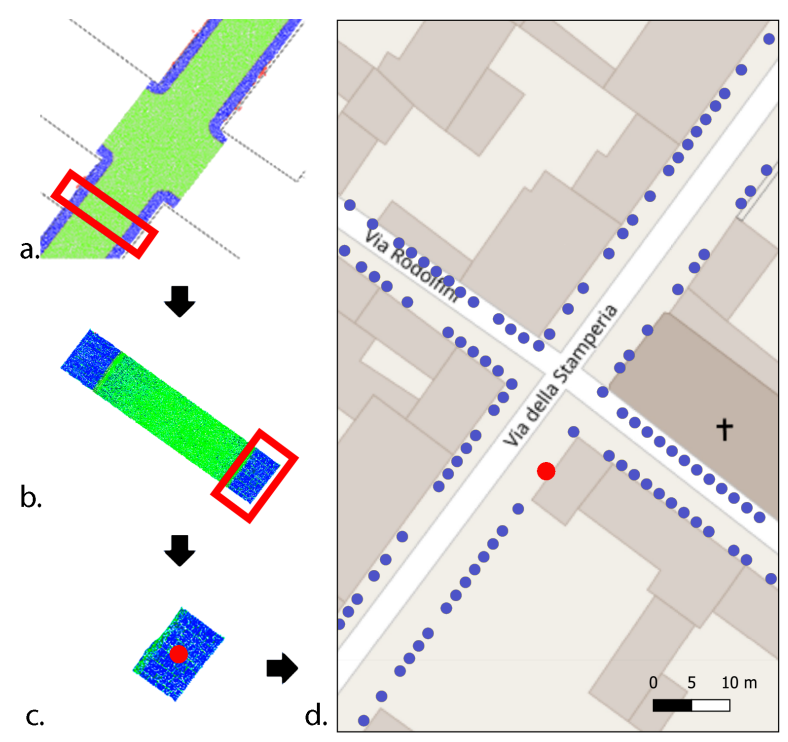

Figure 4. Example of vectorization workflow for a sidewalk segment. Selection of a segment of road on the point cloud (a), selection of the sidewalk cluster of points (b), computation of cluster center (c), and visualization of the cluster center (i.e. node) overlapped to a city map (d). a second one taking in consideration the fact that, in the case study considered, all the streets of the city centre pedestrians can cross the road everywhere (by paying attention to the vehicular traffic, of course). This happens because inside the city centre there are not zebra crossings, so according to Articles 190 and 191 of the Italian highway code, in this situations pedestrians may only cross the road in a perpendicular direction, taking care to avoid situations that endanger themselves or others. The second network has edges that connect sidewalks nodes also crossing the road everywhere; actually considering nodes position, at the moment the crossing in perpendicular was not possible to be added, so when developing the "crossing-theroad" edge, only the ones with shortest length were added to the network.

The route analysis was performed in QGIS using an algorithm made available in the processing toolbox. Among the many tools available under "Processing $>$ network analysis", the one selected for the tests is "Shortest path (point to point)", which was able to compute either the shortest or the fastest path between two points selected directly on the map.

As said, this algorithm could compute the shortest or fastest path. The "shortest" obviously took in consideration only the length of network edges, the "fastest" use the "speed" value of each edge to compute the fastest way to reach the final destination. In this paper, the "fastest path" algorithm was used to compute the most accessible path. By creating a new column in the edges database, and filling it with a fictitious speed (i.e. the speed act as a weight), based on the sidewalk geometric attribute, it was possible to force the algorithm to compute a path that use only the accessible sidewalk edges. For example, considering a sidewalk width of $0.9 \mathrm{~m}$ as accessible (as enforced by Italian laws), and by giving a speed $=1 \mathrm{Km} / \mathrm{h}$ to all edges with "width" $<0.9 \mathrm{~m}$ and speed= $2 \mathrm{Km} / \mathrm{h}$ to all edges with "width" $>0.9 \mathrm{~m}$; it was possible to compute the "fastest" path that actually was forced to be as much as possible on sidewalks with a width which was considered accessible.

As just described, a trick was employed to achieve the aim of calculating accessible routes, using the algorithm that calculates the fastest route and entering a speed value calculated by weighing the accessibility parameter (in this case the width) relative to the pavement. Similarly, the speed value of each edge can be used to reduce the number of crossing-edges used (just by increasing the speed on non-crossing-edges). Considering a path for wheelchair users, crossing-edges should be used only if the $\mathrm{Z}$ difference between road and sidewalk is lower than 2.5 centimetres (to do so crossing edges where difference in $\mathrm{Z}$ is higher should have a very slow speed).

\section{RESULTS}

\subsection{Sidewalks attribute extraction and network creation}

The Sabbioneta point cloud covered the city centre and some areas outside the fortified walls, for a total of more than $6 \mathrm{~km}$. The semantic segmentation was performed for the area inside the city centre, and was described in a previous paper (treccani2021; while the focus of this paper is the data extraction and vectorization.

The sidewalk attributes were computed for all the city centre area analysing the road by segments of 2 meters length. The results of this computation are presented in Table 1, where for 


\begin{tabular}{lllll}
\hline \hline Attribute & Ranges & Most frequent & Reference value & $\begin{array}{l}\text { Percentage of accessible } \\
\text { edges in the sidewalk net- } \\
\text { work }\end{array}$ \\
\hline Width & $0.45 \mathrm{~m} \div 2.20 \mathrm{~m}$ & $0.95 \mathrm{~m}$ & $\geq 0.90 \mathrm{~m}$ & $72.3 \%$ \\
Transverse slope & $0.05 \% \div 9.75 \%$ & $1.25 \%$ & $\leq 1 \%$ & $8.6 \%$ \\
Longitudinal slope & $0.10 \% \div 9.77 \%$ & $2.1 \%$ & $\leq 5 \%$ & $79.3 \%$ \\
Relative Z difference & $0 \mathrm{~m} \div 0.12 \mathrm{~m}$ & $0 \mathrm{~m}$ & $\leq 0.025 \mathrm{~m}$ & $66.5 \%$ \\
\hline \hline
\end{tabular}

Table 1. Results of the sidewalk attribute extraction. The table shows the ranges of values of the attributes together with the most frequent value. The results are then compared with the reference values specified by law.

all the geometric attributes the range of the computed value is presented, together with the most frequent value. Then comparing all values with the reference one (coming from Italian law), it was possible to compute the percentage of sidewalk edges in the network that could be considered accessible.

Subsequently, sidewalks segments spatial position was computed and added to a vector layer (see Fig. 4). The output vector network is composed by 1780 nodes and 1720 edges and was computed in 3.5 minutes on a medium quality computer (16GB RAM, NVIDIA GeForce 950m). Also a second type of edges were computed: considering the possibility of crossing the road everywhere. This second network was composed by the same 1780 nodes, but with 1357 new edges, for a total of 3077. A portion of the two networks can be seen in Figure 5.

After the computation, a manual refinement was necessary to solve some minor errors like few missing edges or some extra edges that needed to be removed. As a measure of the algorithm's performance, only 23 edges needed refinement, which corresponds to $1.3 \%$ of the total, giving an accuracy of $98.7 \%$. Furthermore, considering that the point cloud was covering almost the wall city center, but not all of it, some short roads were not surveyed and for this reason their sidewalks were not in the network. They were added manually.

From the developed network and using the QGIS tools, it was possible to produce several thematic maps that could be used by administrators, planners, but also city users, to get information about sidewalks attributes. As an example, Figure 6 shows the accessible (in green) and inaccessible (in red) sidewalk segments according to their width. In fact, for Italian law sidewalks are accessible if their width is higher than 0.9 metres.

\subsection{Path computation}

Path computation was tested on two different cases. In the first case study, either the shortest path and the fastest path were computed considering sidewalks width. In the second test the presence of ramps on sidewalks with higher elevation respect road was considered in the path computation.

The first test is provided in Figure 7. In this example a path from a generic area of the city (point $\mathrm{A}$, a crossing between two roads) is computed towards a POI of the city: the Galleria degli Antichi (point B). In Figure 7b the shortest path is computed; while in $7 \mathrm{c}$ the fastest path considering accessibility (width) is computed. For this second case, a different speed was given to sidewalk with a width considered accessible $(4 \mathrm{Km} / \mathrm{h})$, and the ones considered inaccessible $(0.001 \mathrm{Km} / \mathrm{h})$. Given the big difference between the two speeds, when considering the fastest path, the algorithm avoided as much as possible the sidewalks with an inaccessible width. In fact, it can be noticed (comparing the predicted path in $7 \mathrm{c}$ with the thematic map in $7 \mathrm{a}$ ) that

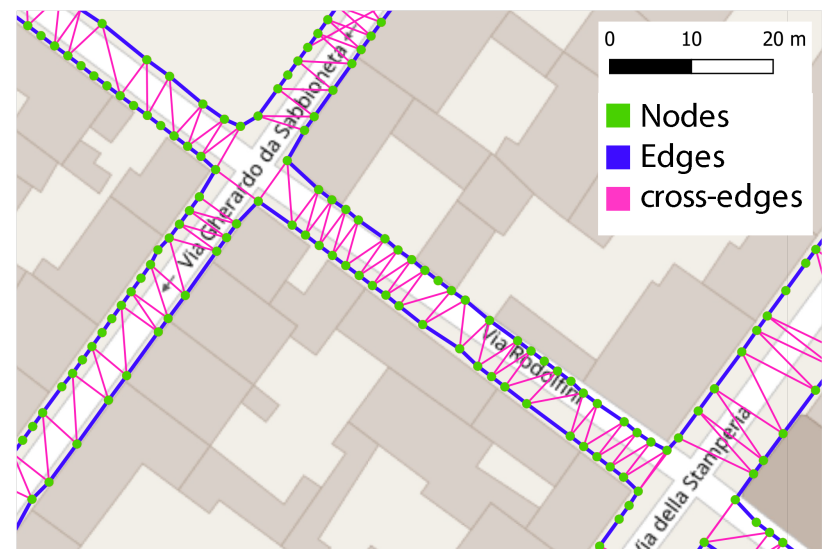

Figure 5. A portion of the city of Sabbioneta, green circles are the nodes, representing the sidewalks segments, blue lines are the edges connecting the sidewalks along the sidewalk path, and purple lines are edges that connects sidewalk nodes crossing the road, considering the possibility for pedestrian to cross everywhere inside the historic center of the city.

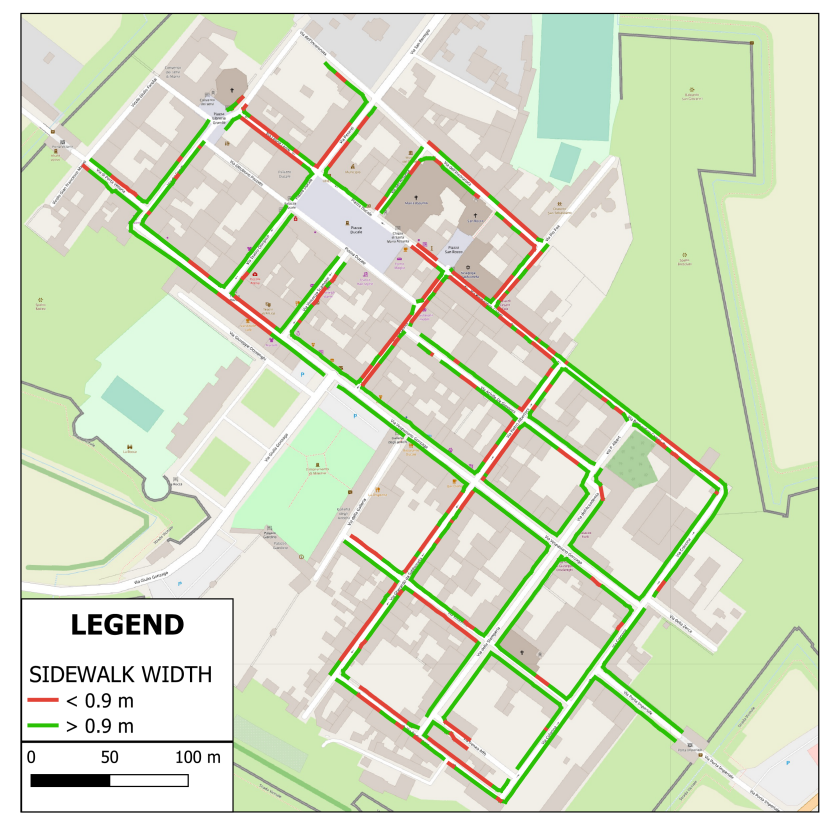

Figure 6. Thematic map of the entire city of Sabbioneta developed using the computed network edges and their attributes. In green sidewalk segments with width $>0.9 \mathrm{~m}$ (considered accessible by Italian law) and in red with width $<0.9$ m (not accessible). 

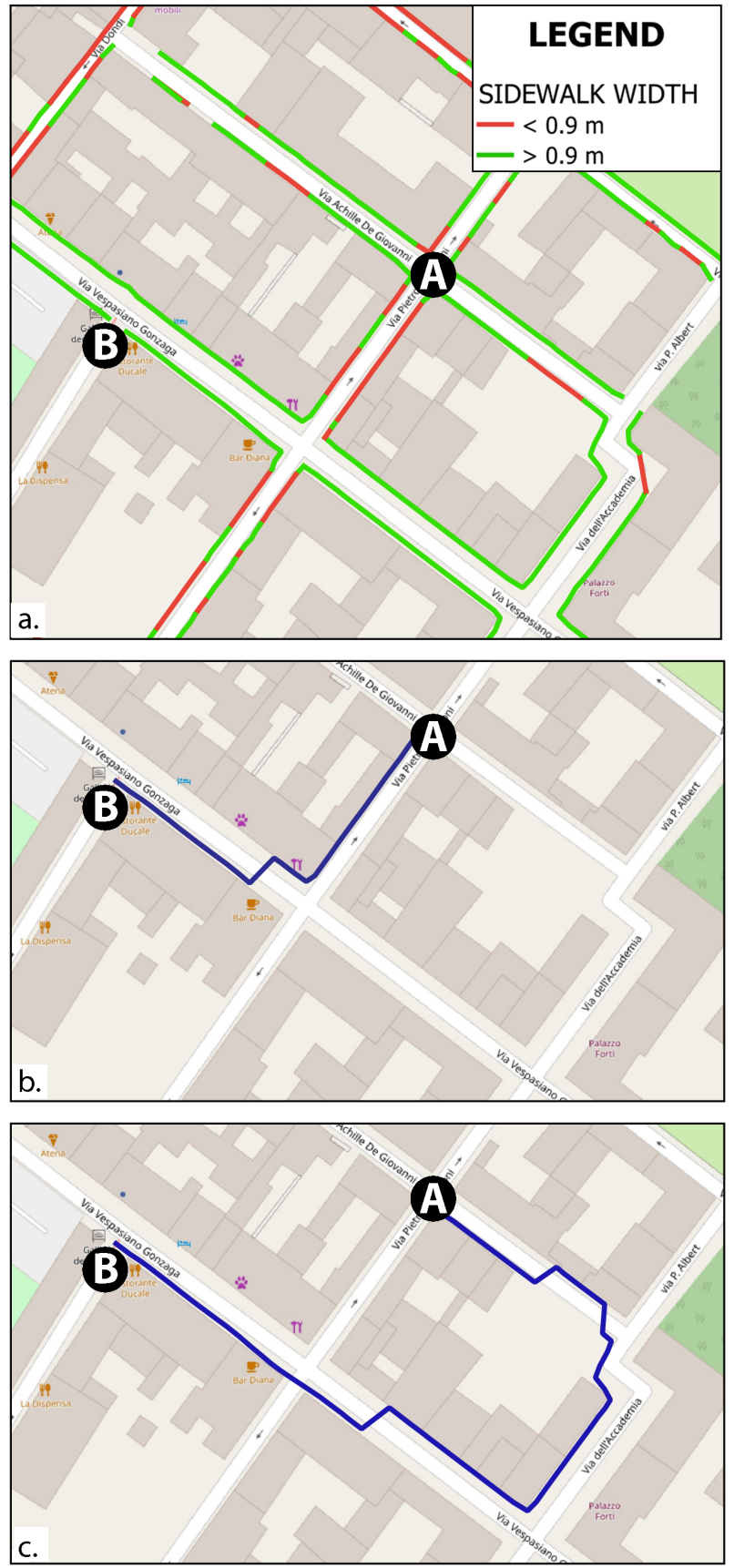

\section{$01020 \mathrm{~m}$}

Figure 7. Test 1: computation of path considering width of sidewalks as a constraints. The path is computed from point A to point B. (a) sidewalk width thematic map; (b) shortest path; (c) fastest path considering accessible width.

the suggested path avoid to use a small portion of inaccessible sidewalk in the top right corner, and for this reason crossed the street only for few meters.

The second test is provided in Figure 8. In this case, the path from Piazza Ducale (point A) to the Galleria degli Antichi (point B) was computed. The route was computed considering the different elevation of sidewalks respect the road, making impossible for wheelchair users to cross the road everywhere.
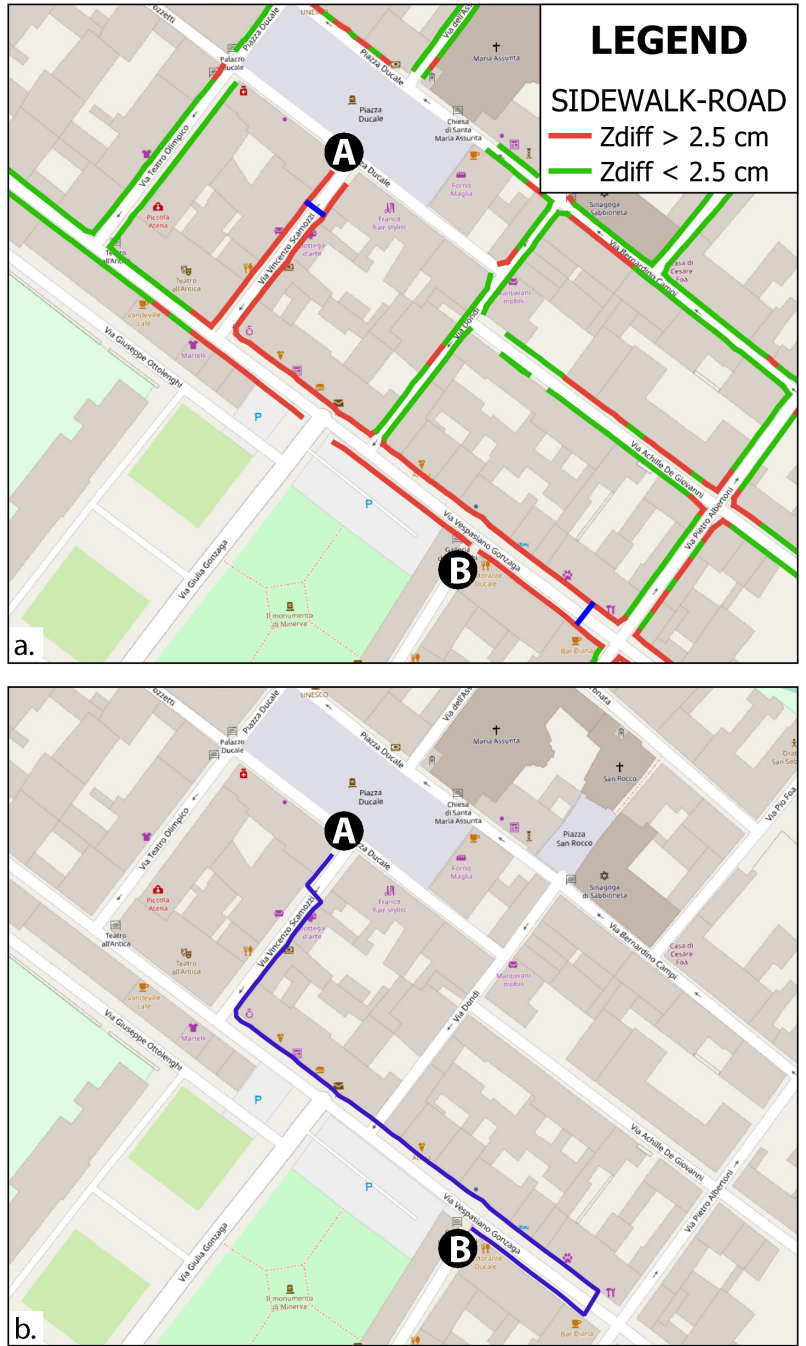

$01020 \mathrm{~m}$

Figure 8. Test 2: computation of path considering $\mathrm{Z}$ difference between sidewalks and roads as a constraints. The path is computed from point A to point B. (a) sidewalk Z-difference thematic map; (b) shortest path considering also the presence of ramps on sidewalks.

As it is possible to see in Figure 8a, the sidewalks that connect point $\mathrm{A}$ to point $\mathrm{B}$ are all characterized by a difference in $\mathrm{Z}$ between road and sidewalk which is higher than the acceptable value (which for Italian law is $2.5 \mathrm{~cm}$ ). To allow the path computation in this case it was necessary to add to the network two extra edges, in correspondence of ramps on sidewalks. The two added edges can be seen in Figure 8a (in blue, close to point A and on right respect point B). As provided by Figure 8b, the computed path was using the two ramps to overcome the inaccessibility issue of sidewalk elevation.

\section{DISCUSSION AND CONCLUSIONS}

This paper presented a method to automatically compute accessible routes within an historic sites starting from point clouds data. The accessibility was tackled by exploiting geometric attributes of sidewalk network and comparing them with Italian law. The starting point of the work was a semantically segmen- 
ted point cloud, whose points were classified in road and sidewalk. Sidewalks were then subdivided in segments of 2 metres length and some of their geometric attributes were extracted (width, elevation respect road, slopes, paving material). Then they were vectorized to create nodes for a network. Nodes were regularized considering contextual information (position and shape of crossings between roads) derived from OSM. Nodes were then connected by edges following two approaches: connecting nearest sidewalks and connecting sidewalks by crossing the street. The second approach was motivated by the fact that in the case study selected, but also in many other cases, there were not zebra crossings, so pedestrian are allowed to cross the street everywhere.

Sidewalk attributes extraction was performed following simple formulations, the results provided in Table 1 shows that the computed value have reliable ranges. For all the attributes a refinement was implemented to take care of noisy points in the point cloud, for example widths higher than 10 meters means that there was an issue in the computation. In these cases the value were manually refined or removed. It is noticeable that extracted sidewalk attributes, compared with reference values, show that for the most part of the city the transverse slope limit is not respected, this is an important issue that should be later investigated, specifically a more refined method for the computation of transverse slope could be implemented.

The result shows that the automatic vectorization of sidewalk nodes and attribute extraction has an accuracy of $98.7 \%$, which means that only a minor manual refinement was performed at the end of the process. Of course, a limit of this method is that it only was able to vectorize sidewalks that were surveyed by the MLS system, so roads that were not surveyed must be added manually. Despite some minor roads, almost the entire city sidewalk network was correctly vectorized and the sidewalks attributes were used to produce thematic maps that can be suitable for future a future assessment of the sidewalks conditions, but also to help administrators and planners in the decision making process.

For the routes computation, the path computation tool preinstalled in QGIS was used. It allowed to compute the shortest path, along the sidewalk network, between two point selected on the map. It also allows to compute the fastest path considering a speed; in the paper this second algorithm was implemented to compute the accessible path by acting on the speed value of each edge as a weight. In the paper two tests were made, considering a weight to the sidewalk width and a weight to the sidewalk elevation respect road. In both the cases the algorithm was able to predict a path that was passing only on sidewalks considered accessible for the specific attributes selected for the weight. Referring to the first test, two different speeds were implemented: $0.001 \mathrm{Km} / \mathrm{h}$, and $4 \mathrm{Km} / \mathrm{h}$, it is important to notice that with a smaller difference in speed (e.g., $1 \mathrm{Km} / \mathrm{h}$ and $2 \mathrm{Km} / \mathrm{h}$ ) the result of the algorithm would not have been satisfactory. The big difference between the two speed ensured that the computed path was completely accessible.

The path computation here was performed implementing an existing tool and acting on the "speed" value to obtain the result. The speed value was weighted depending on one accessibility feature of sidewalk edges. In future works a possible combination between several weights coming from all the geometric attributes will be considered. The possibility given by other existing path computation algorithms and also by a tailor made one will be investigated.
In light of the presented results, some possible improvements to the method could be the exploiting of presence of porches: in fact the routing could be computed on the basis of people behaviour in different weather conditions (sunny, rainy) using mostly covered areas instead not covered ones for their trip between POIs. A useful improvement of the method could be the automatic detection of ramps and zebra crossings, instead of inserting them manually at the last stage. In Literature there are methods to detect zebra crossing or ramps, and should ideally be implemented easily in the method.

The method was tested for a MLS point cloud, so the trajectory data was available, but, alternatively, the road centerline from OSM or cadastral database could be used, making the method applicable also with point clouds acquired with other instruments (e.g., drones, Terrestrial Laser Scanning).

In future works the extracted paths and vector layer could be used for the development of an interactive webGIS to be used by many city users (citizen and tourists) and by administrators and city planners. also investigating some special scenarios: some roads closed at vehicular traffic and only used for pedestrian, accessible paths considering sidewalk attributes. The routing algorithm, together with the vector maps, could also be implemented in an application for mobile phones.

\section{ACKNOWLEDGEMENTS}

The authors wish to thank Leica Geosystem Italy for the support provided during the test with instrument Leica Pegasus:Two. The second author has received fundings through human resources grant RYC2020-029193-I funded by MCIN/AEI/10.13039/501100011033 y FSE El FSE invieste en tu futuro.

\section{REFERENCES}

Hou, Q., Ai, C., 2020. A network-level sidewalk inventory method using mobile LiDAR and deep learning. Transportation Research Part C: Emerging Technologies, 119, 102772. https://doi.org/10.1016/j.trc.2020.102772.

Li, H., Cebe, J., Khoeini, S., Xu, Y. Dyess, C., Guensler, R., 2018. A Semi-Automated Method to Generate GIS-Based Sidewalk Networks for Asset Management and Pedestrian Accessibility Assessment. Transportation Research Record, 2672(44), 1-9. https://doi.org/10.1177/0361198118757981.

Luaces, M. R., Fisteus, J. A., Sánchez-Fernández, L., MunozOrganero, M., Balado, J., Díaz-Vilariño, L., Lorenzo, H., 2021. Accessible Routes Integrating Data from Multiple Sources. ISPRS International Journal of Geo-Information, 10(1). https://www.mdpi.com/2220-9964/10/1/7.

Marconcini, S., Pracchi, V., 2019. Inclusive Cultural Heritage sites: ICT as a tool to support the design process and share knowledge. The International Archives of the Photogrammetry, Remote Sensing and Spatial Information Sciences, XLII2/W11, 793-800. https://www.int-arch-photogramm-remotesens-spatial-inf-sci.net/XLII-2-W11/793/2019/.

Marconcini, S., Treccani, D., Díaz-Vilariño, L., Adami, A., 2021. A data collection framework for managing accessibility and inclusion in urban heritage. ISPRS Annals of the Photogrammetry, Remote Sensing and Spatial Information Sciences, VIII-M-1-2021, 101-108. https://www.isprs-ann-photogrammremote-sens-spatial-inf-sci.net/VIII-M-1-2021/101/2021/. 
Pella, H., Ose, K., 2018. Network Analysis and Routing with QGIS. John Wiley Sons, Ltd, chapter 4, 105-144.

Saha, M., Saugstad, M., Maddali, H. T., Zeng, A., Holland, R., Bower, S., Dash, A., Chen, S., Li, A., Hara, K., Froehlich, J., 2019. Project Sidewalk: A Web-Based Crowdsourcing Tool for Collecting Sidewalk Accessibility Data At Scale. Association for Computing Machinery, New York, NY, USA, 1-14.

Treccani, D., Díaz-Vilariño, L., Adami, A., 2021. Sidewalk detection and pavement characterisation in historic urban environments from point clouds: preliminary results. The International Archives of the Photogrammetry, Remote Sensing and Spatial Information Sciences, XLIIIB4-2021, 243-249. https://www.int-arch-photogramm-remotesens-spatial-inf-sci.net/XLIII-B4-2021/243/2021/.

Verde, D., Silva, T., Pereira, A. I., Paiva, S., 2021. Optimized routing for people with permanent or temporary mobility disability: a case study in viana do castelo. 2021 IEEE International Smart Cities Conference (ISC2), 1-6. 